\title{
An Architecture Supporting Intelligent Mobile Healthcare Using Human- Computer Interaction HCI Principles
}

\author{
Mesfer Alrizq ${ }^{1}$, Shauban Ali Solangi ${ }^{2}$, Abdullah Alghamdi ${ }^{1, *}$, Muhammad Ali Nizamani $^{2}$, \\ Muhammad Ali Memon ${ }^{2}$ and Mohammed Hamdi ${ }^{1}$ \\ ${ }^{1}$ College of Computer Science and Information Systems, Najran University, Najran, Saudi Arabia \\ ${ }^{2}$ Faculty of Engineering \& Technology, University of Sindh, Jamshoro, 78060, Pakistan \\ ${ }^{*}$ Corresponding Author: Abdullah Alghamdi. Email: abdulresearch@hotmail.com \\ Received: 22 March 2021; Accepted: 12 May 2021
}

\begin{abstract}
Recent advancements in the Internet of Things IoT and cloud computing have paved the way for mobile Healthcare (mHealthcare) services. A patient within the hospital is monitored by several devices. Moreover, upon leaving the hospital, the patient can be remotely monitored whether directly using body wearable sensors or using a smartphone equipped with sensors to monitor different user-health parameters. This raises potential challenges for intelligent monitoring of patient's health. In this paper, an improved architecture for smart mHealthcare is proposed that is supported by HCI design principles. The HCI also provides the support for the User-Centric Design (UCD) for smart mHealthcare models. Furthermore, the HCI along with IoT`s (Internet of Things) 5-layered architecture has the potential of improving User Experience (UX) in mHealthcare design and help saving lives. The intelligent mHealthcare system is supported by the IoT sensing and communication layers and health care providers are supported by the application layer for the medical, behavioral, and health-related information. Health care providers and users are further supported by an intelligent layer performing critical situation assessment and performing a multi-modal communication using an intelligent assistant. The HCI design focuses on the ease-of-use, including user experience and safety, alarms, and error-resistant displays of the end-user, and improves user's experience and user satisfaction.
\end{abstract}

Keywords: Human computer interaction; mhealthcare; user-centric design; sensor network; nternet-of-things

\section{Introduction}

Smart technologies are everywhere in the form of cloud computing, edge computing, distributed computing, mobile computing, and IoT (Internet of Things). These technologies paved the way for the advancement of healthcare devices [1]. The IoT is an environment of a variety of things, such as RFID tags, medical devices, mobile phones, etc. Such devices connect through unique identifiers and interact with each other [2,3]. IoT connected devices transfer data between each other, in turn leading to new derived data. Health care is one of the most important application areas of IoT. It provides opportunities

This work is licensed under a Creative Commons Attribution 4.0 International License, which permits unrestricted use, distribution, and reproduction in any medium, provided the original work is properly cited. 
for several medical applications, such as mobile and remote health monitoring. The integration of wearable devices and systems in IoT help providing better mhealth services. The significant and potential betterments resulting in improved healthcare interventions. Mobile health care or the mHealthcare devices and applications potentially give the advantage of recording and retrieving health-related information ranging from fitness levels and heart rates to medication dosages and sleep cycles. However, mHealthcare is essential for user and healthcare service providers. In critical cases, if the healthcare worker is away, the users can monitor their health conditions. On the other hand, the HCI is essential for mHealth devices, which in turn provides the interpretation of dissemination of health services and health data. The HCI supports the ease and efficient usage of these devices for both health care professionals (HCPs) and patients [3-5]. The HCI with good design is a key to provide smart mobile healthcare and mHealth environment, with long term health monitoring and tracking solutions. This HCI-based mHealth monitoring system lays down the rules for timely and quick response for emergency and the elderly patient care a the mHealth care centers [6-8]. The HCI perspective for smart mHealthcare system design reflects that the HCI along with IoT cloud-based mHealthcare environment is critical due to provision of usability and usefulness, as shown in Fig. 1. In addition, the design eschews the defects in the IoT devices. Within the mHealthcare, the environment determines the effectiveness of the system to detect the problems, identifies state-of-the-art solutions to the problem, and outlines priorities and resource allocation for the betterment of health results $[9,10]$.

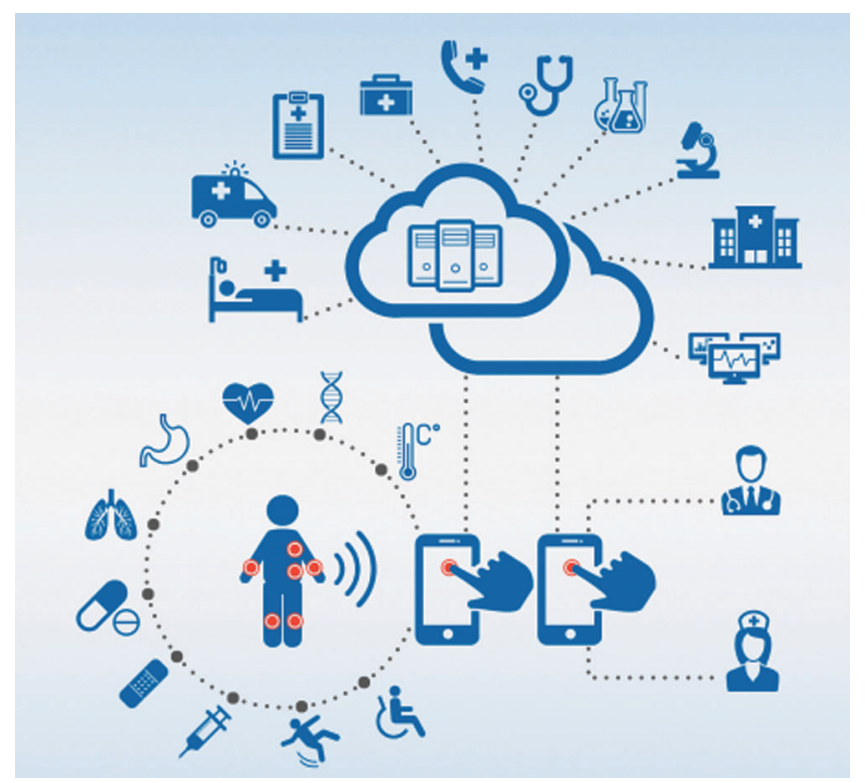

Figure 1: Smart mHealthcare environment based on HCI and IoT [11]

Therefore, we propose an HCI smart mHealthcare model that is principled to complement the usercentered design method and satisfaction of the end-user in order to provide a better user experience. This promotes the development of these devices with smoother integration in order to facilitate efficient connections among smart mHealthcare, devices, HCPs, and users [11].

The HCI issues, related to the design for usability from both the user side and healthcare providers, comprise several design challenges. Those design challenges might be the poor design of such devices, unawareness of the system, and compatibility of these devices. Smart mHealthcare design mitigates a poor usability barrier, which is considered as a major adoption for mHealthcare applications. However, the IoT devices' compatibility plays an inevitable part in overall HCI design, in which users get 
sophisticated interaction with the system. Consequently, the HCI design specifically incorporates all required key points to address UCD (User Centric Design) application [11,12]. The UCD design encapsulates major benefits in a smart mHealthcare environment. It ensures the usability of the application and provides the necessary information in a complex environment. Both the user (patient) and healthcare service providers can get in touch on an immediate basis. The system can also support multiple users and provide their health-related information HCPs. This demands a realistic and wholesome efficiency of the system. Furthermore, the smart mHealthcare environment assures timely data delivery, good performance of the system. The robustness of the smart mHealthcare system leverages the potential benefits in the reduction of complexity in the HCI environment. The smart mHealthcare HCI design is based on the UCD mitigate the reliance and reluctance on limited healthcare infrastructure and speed up the healthseeking practice [13]. This is why good care and better health results become the ultimate goal of the overall healthcare environment. Therefore, a smart mHealthcare HCI design should fulfill the goal of a good user experience with a robust system for both the users (patients) and doctors. The HCPs can monitor and diagnose the patients that have chronic health conditions namely heart diseases, diabetes, obesity, and elderly patients. The HCPs can keep track of any important change in a monitored condition and communicate effectively and quickly with the patient [14].

The rest of the paper is organized as follows. Section I provides the introduction to the HCI and smart IoT systems. Section II describes the literature review with existing and current research. Section III incorporates the smart HCI mHealthcare architecture. Section IV explains the suggested model of HCI smart mHealthcare for medical devices. As a final point, a conclusion and future work is provided in Section V.

\section{Literature Review}

Traditionally, research about medical device design is mostly related to usability and ergonomics as these are important for approval from the regulatory bodies. These types of information communication and technological applications in medical science paved the way for more complicated devices, so HCI design stressed the interaction between computer systems and HCPs [15-17]. Nowadays, smart technologies have led to a substantial change in employing and designing medical devices. From the smart technology perspective, the mHealthcare services have been transformed into personalized care delivery with the help of sensor technology, cloud computing, mobile computing, edge computing, big health data, social networks, mobile and wireless communication technology, and health apps $[18,19]$.

A new healthcare model is, undoubtedly, imminent for service delivery, and centers on the users and smart healthcare services. The model with ten Ps for medicine defines the Personalized, Perspective, Predictive, Preventive, Precise, Participatory, Patient-Centric, Psycho-Cognitive, Post-Genomic, and Public [20]. Accordingly, it is necessary for both HCPs and patients or users that smart devices are the main component for the implementation of mHealthcare services and models. The study in Fanos [21] claims that the biggest IT and internet companies of the world are looking for prospective opportunities and domains for the investment into the medical field and healthcare services, based on expertise in IoT, AI (Artificial Intelligence), and cloud computing or mobile computing. Also, it is expected that the mHealthcare market will reach more than 63 Billion US\$ by the end of 2021. IoT-based mHealthcare devices and market adoption will further grow up to 410 Billion US dollars. The HCI provides the main support for data visualization, comprehension, UI control in the IoT mHealthcare domains [22]. In Solanas et al. [23,24] scholars emphasized that smart mHealthcare devices provide promising results and help ease in managing asthma, diabetes, loss of hearing, depression, poor sightedness, migraines, anemia, and osteoarthritis. 
Smart healthcare systems and mobile devices can provide better and robust results unitedly in the areas of treatment, rehabilitation, diagnosis, and measures of prevention. Further, smart mHealthcare enhances the HCPs and patient's interactions. The interaction for the elderly or chronic diseases patients has been increased to a great extent with collaborative care, enhanced information of patients using data based on current conditions. This improves the system's effectiveness in terms of usability and efficiency for HCPs and patients [25]. In Martínez-Pérez et al. [26-28], the authors used mobile and wireless communication technology for the healthcare services for the low cost and improved service of healthcare with flexibility and efficiency. Remote advancements give more noteworthy adaptability and convenience contrasted with customary arrangements [29] and the capacity of savvy gadgets to share data all the more effectively gives the chance of far-off finding [30-32]. HCI helps the new advancements to be promptly accessible, available, adaptable, and adequate [33]. In addition, it assists with guaranteeing that arrangements address client issues and convey viability and effectiveness [34-37].

The studies in Webb et al. [38] suggest that smart healthcare services need the real-time processing, sharing, and utilization of precise data. Thanks to cloud computing and wireless communication technologies for providing seamless services and playing key roles in service delivery, sensor network, and people. They provide details of major applications of smart healthcare systems for patient monitoring, patient data analysis, and collection for smart and intelligent decision-making processes. Along With these services, telemedicine and personalized medical services provide smart and mobile healthcare services. In Fairbanks et al. [39], mobile WBAN (Wireless Body Area Networks) was used and claim efficient healthcare delivery and promise low-cost prevention and management of the patient-centered disease. Their research focuses upon the acceptance, usability, and effectiveness, and efficiency highly demands the HCI design considering the human characteristics depending on technological and social backgrounds. It, however, does not focus much on patient situation assessment and intelligent assistance. Moreover, it does not focus on improving the user experience and parameters affecting the adoption of the system by both the health care providers and end-users. This is an important factor for the low adoption rate of the current technologies [40-43]. Thus, there is a need for intelligent mHealthcare innovation and user acceptability with multidisciplinary collaboration among HCPs, HCI, and health experts $[44,45]$.

\section{Smart mHealthcare based IoT Architecture}

Smart mHealthcare is the combination of Information Technology (IT) and life sciences for the provision of better healthcare services and cost-effectiveness. Initially, IBM originated a strategy called Smart Planet with suggestions to embed sensors into a diversity of physical objects. These objects are connected and integrated through cloud computing [46-53]. The main application of this technology is to facilitate HCPs, users/patients and establish e-Health records (electronic health records) for the intelligent monitoring and tracking of patients. This system helps to build a sustainable ubiquitous system for smart mHealthcare and will boost service delivery for better patient care. We suggest a design architecture with five layers for a smart mHealthcare environment one additional layer, which is the AI layer for situation assessment using predictive modeling and intelligent assistant, as shown in Fig. 2, with each layer depending on the subsequent layer. It enhances the four-layered architecture, as proposed in Liu et al. [11]. Other layers include the Application layer, Data Integration Layer, Communication Layer, and Sensing Layer.

The 5-layered design for the smart mHealthcare environment chiefly advocates the mHealthcare scenario where the Application layer incorporates dashboards, a system for outbreak or epidemic systems, platforms for healthcare at regional level, personal health records, and smart monitoring and tracking system [54,55]. The application layer utilizes the websites, dashboards, IoT devices, and applications as shown in Tab. 1. The second layer is the AI layer, which performs patient situation assessment, which may involve integrating temporal events and communicating using multimodal dialog. The third layer is 
the data integration layer, which deals with resources for the medical data of patients and doctors or HCPs information, cloud computing platforms, distributed data storages, data fusion, information sharing, data processing, predictive modeling, data analysis with the help of IoT applications and devices. The fourth layer is the communication layer, which is responsible for all telecommunication services and access, fundamental IoT layer. Wireless networks and broadcasting networks use state-of-the-art technologies to provide telecommunication facilities, as shown in Tab. 1. The sensing layer utilizes sensor technologies along with wireless technologies for the purpose of gatheing the information from the vicinity [4,56-58]. The sensors may include continuous monitoring sensors such as ECG, EEG, EMG sensors, gyroscopes, accelerometers, auditory, and visual sensors. Discrete-time data collection sensors are there such as blood pressure, blood oxygen saturation, humidity \& temperature sensors, and glucose monitoring sensors. These sensors may use Bluetooth/ZigBee, RFID, or Ultrawide Band (UWB) wireless technologies to communicate with the network, as described in Tab. 1.

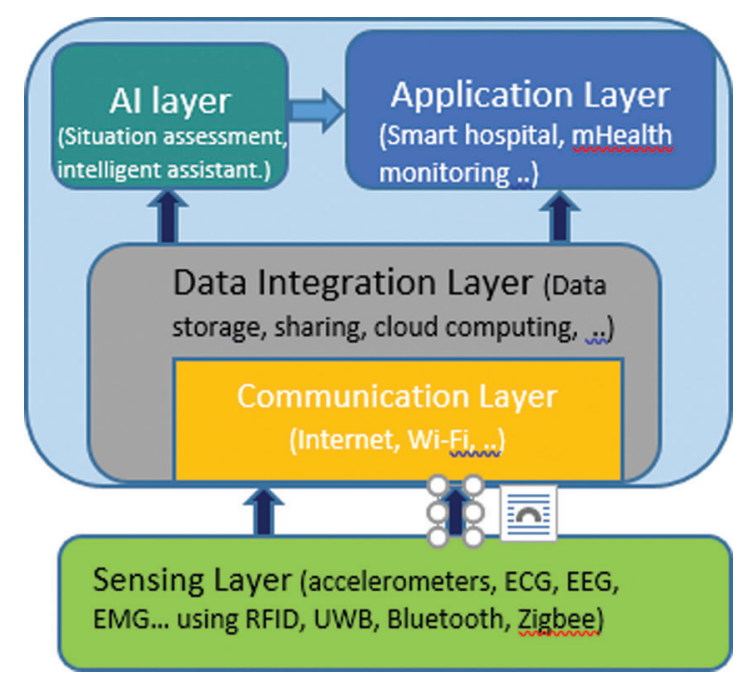

Figure 2: Smart mHealthcare 5-Layer architecture

Table 1: Smart mHealthcare 5-layer information architecture

\begin{tabular}{|c|c|}
\hline Layers & Application and implementation \\
\hline $\begin{array}{l}\text { Application } \\
\text { Layer }\end{array}$ & $\begin{array}{l}\text { Remote patient monitoring, health records storage, elderly patients tracking and } \\
\text { monitoring, Locate the physicians in the area as well as in the hospital. Smart health } \\
\text { monitoring, home health system, epidemic outbreak detection system. Mostly, the } \\
\text { delivery mode is in the form of dashboards, websites, e-Medical Records }\end{array}$ \\
\hline AI layer & Patient situation assessment, chronicle recognition, intelligent assistance \\
\hline $\begin{array}{l}\text { Data Integration } \\
\text { Layer }\end{array}$ & $\begin{array}{l}\text { Data integration and timely sharing, cloud platforms, distributed computing, data } \\
\text { extractions, data fusion, statistical analysis and data analysis. }\end{array}$ \\
\hline $\begin{array}{l}\text { Communication } \\
\text { layer }\end{array}$ & Internet, wireless networks, Information and communication systems \\
\hline Sensing Layer & $\begin{array}{l}\text { Sensors: Continuous time varying (ECG, EEG, EMG, Accelerometer, gyro, camera, } \\
\text { mic..), Discrete Time Varying (Temperature, humidity, BP, Blood } \mathrm{O}_{2} \text { saturation, } \\
\text { Gulocose..), } \\
\text { Sensor technology: UWB, RFID, BAN, Wearable, ZigBee/Bluetooth, }\end{array}$ \\
\hline
\end{tabular}




\subsection{Application Layer}

This layer is responsible for HCPs decision making and helps to communicate with the user/patient. Also, it helps in the evaluation and realization of e-records (electronic records) and smart mHealthcare requirements. Many types of health-related applications and keeping up-to-date e-records are maintained and monitored. Therefore, the security provider must be aligned with the utilization of precise and adequate information sharing among patients and HCPs. For accessing the specific information and medical resources, the application layer provides HCPs and patients with the ease and efficient links and connections of smart mHealth resources and service [59]. The application layer gives an access to services namely main spots of smart mHealthcare service to the patients and HCPs, management authorities and decision-makers, and institutional bodies. The decision-maker includes drug oversight bodies, Health Departments and national healthcare centers, public health departments. The institutional health bodies comprise hospitals, community clinics, regional health centers, and nursing homes [60].

\subsection{AI Layer}

The AI layer incorporates mechanisms for intelligent decision-making and assistance. This layer embodies situation assessment using predictive modeling, especially focusing on the recognition of chronicles, i.e., temporal events. The patient's critical parameters, such as blood oxygen level can drop silently (silent hypoxia), without showing any external symptoms of shortness of breath or difficulty breathing. This layer is critical in terms of assessing patient situations, recognizing a course of action, and communicating with the both health care provider and the user. The communication can be multimodal in the form of an intelligent (virtual) assistant. This layer is supported by the data integration layer and will support the application layer.

\subsection{Data Integration Layer}

The Data Integration layer helps to accomplish data processing and data fusion. They are extremely basic but important requirements for the support of smart mHealthcare services. This layer utilizes service-oriented architecture, cloud computing technologies, and big data to build an e-inventory for health, where records are kept and shared with the corresponding personals. This augments the collected data with the data seeking from other smart systems. Overall, integrated data works to support the application layer platform.

\subsection{Communication Layer}

This layer facilitates both the patients and HCPs with communication services. The communication services along with body sensor networks, internet, and WiFi services combining with the optical fiber to provide very high-speed and reliable internet connectivity. Without communication service and infrastructure, the smart mHealthcare system are not viable. The communications networks provide huge capacity, robust internet facilities, and storage of huge amounts of data and sharing the information using internet hubs among the HCPs and patients [61]. The smart mHealthcare environment demands real-time, reliable, and secure data transmission. Thus, telecommunication infrastructure must be well designed and robust to meet the smart mHealthcare needs.

\subsection{Sensing Layer}

It is the most important and fundamental layer of the architecture, so it performs the most important task, which is the collection of health-related information from the patient, whether in the hospitals, in the elderly care centers, or at home. This layer is at the core of IoT technology and is supported by biotechnology. This layer, using a wearable sensor network, helps in gathering the data related to the patient health. The various health parameters can be monitored, such as patient blood pressure, blood oxygen saturation, temperature, 
blood sugar level, ECG, electroencephalograph (EEG), electromyography (EMG), heart-beat monitoring, and visual monitoring. The underlying technology of data transmission depends on the required power consumption, ranging from Bluetooth/ZigBee to ultra-wideband (UWB) wifi communication. The main communication layer receives this data and can directly transmit it to HCPs (using some application to monitor) via the internet or store at a cloud platform (data integration level) [62].

\section{HCI and Smart mHealth Design for Devices}

The HCI design needs to incorporate the ease of use, including user experience and safety, patient and HCPs relationship, legacy support, distinguish end-user, alarms and error resistant displays, timely responses, personalization, and privacy, as illustrated in Fig. 3. The design must ensure the satisfaction of the user and the reliability of the device for the accomplishment of the task. Because every individual user may have a different task to perform, the HCI design of the device must meet the requirement in terms of different user roles, workflows, system functions and dynamic environments, reception storage, real-time information transmission, and data availability.

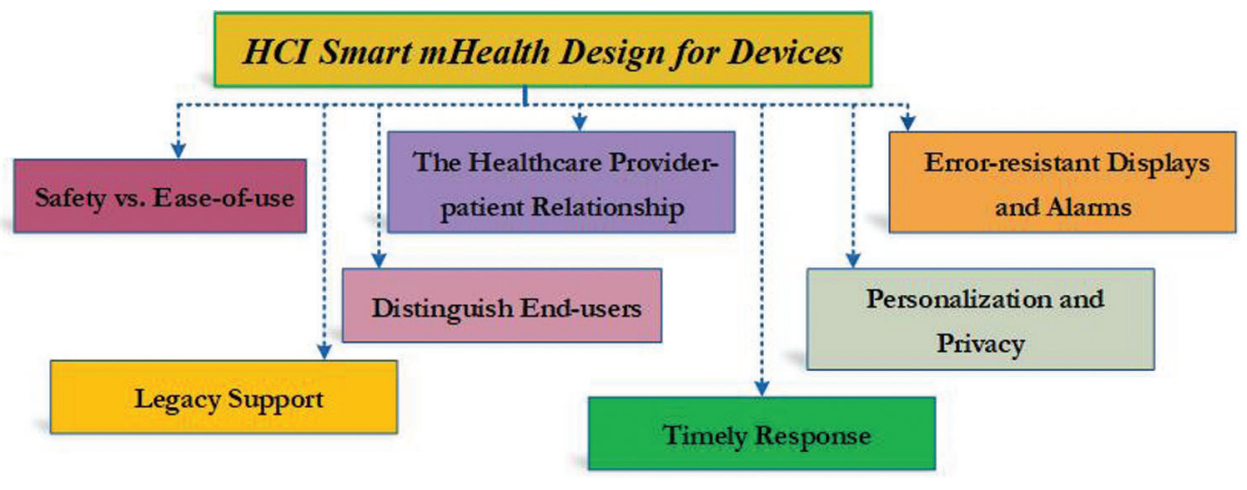

Figure 3: HCI Smart mHealth model for devices

Safety is the foremost consideration in the design principle for the HCI smart mHealthcare system because usability must ensure reliability, satisfaction, effectiveness, efficiency, and secure information utilization as well as accessing. The heuristic evaluation principles must be incorporated [63]. In the case of medical data and devices, the heuristic evaluation may create conflict with ease of use with the safety of the device, as shown in Fig. 3. However, the ease of use motivates us to diminish the cognitive load by use of cues to perform well or even automatically, which needs a very cautious task check. This automatic response is also known as muscle memory and response chaining. Therefore, this type of behavior is undesirable for safety and critical to medical devices.

The manufacturer of the device and the device user expect or intend the errors and omissions in the result of the device. Therefore, errors can occur if safe usability is violated. The usability errors are related to HCI design and UI design. If we take an example of the error in the visual display of UI in the devices installed by the FDA (Food and Drug Administration) Department of US, where, the software was poorly designed that made the doctors confused to visualize the right and left hemispheres of the patient's brain resulting in wrong side surgery of the brain. Therefore, false alarms play their part number one hazards of health technology [64-68]. The false alarms interrupt doctors amid the critical tasks. The false alarms not distract the doctors and make them fatigue but also create noise which increases stress on HCPs and the patients [69-73]. So the HCI designer can design a better user interface and help in preventing and reducing usability errors. The prevention of the errors can be achieved by an iterative usability test, as indicated in Tab. 2. This also helps for analyzing contextual risk during the actual use. 
Table 2: HCI Smart mHealthcare design methods

\begin{tabular}{|c|c|}
\hline HCI Design Method & Specification \\
\hline Evaluate in Use & $\begin{array}{l}\text { The usability evaluation is to evaluate user interaction with the device or } \\
\text { model, user experience. To develop iterative development and design. The } \\
\text { overall aim is to optimize and create ease in user experience and usability } \\
\text { according to the user's needs and criteria. }\end{array}$ \\
\hline Develop \& Implement & $\begin{array}{l}\text { In the development phase, device design meets the application layer criteria } \\
\text { and the device must ensure good performance. The implementation of the } \\
\text { device must be compatible with other devices and sensors network. From } \\
\text { application layer to data integration layer through sensing layer and } \\
\text { communication layer device's development and implementation meet the } \\
\text { needs and compatible and able interconnect and communication. }\end{array}$ \\
\hline $\begin{array}{l}\text { Design Solutions } \\
\text { Evaluation }\end{array}$ & $\begin{array}{l}\text { Design evaluation through the best solution is the foremost need. An iterative } \\
\text { model and redesigning improves the solution design in each phase } \\
\text { prototyping and moving from low-fidelity to high-fidelity. For HCI's best } \\
\text { design, the iterative model follows some steps namely planning and analysis, } \\
\text { specification of ideas, and modeling. This leads to building a prototype and } \\
\text { design evaluation. }\end{array}$ \\
\hline Produce Prototypes & $\begin{array}{l}\text { Building a good prototype of the HCI application design is suitable for the } \\
\text { user interface. } \\
\text { The design includes the user experience by the content of the application, } \\
\text { behavior, and form of the design. Thus, the prototype with a good content } \\
\text { design, interaction model, and visual design provide the satisfaction of the user. }\end{array}$ \\
\hline Produce Design Solutions & $\begin{array}{l}\text { The design solution production related to workflow, task, and content of the } \\
\text { information architecture results in optimized and smart HCI architecture. This } \\
\text { result is the outcome of the previous phases of the iterative design. To } \\
\text { integrate the information with both layers sensing layer and data integration } \\
\text { layer, data acquisition must be compatible and relevant resources. }\end{array}$ \\
\hline $\begin{array}{l}\text { Specification and } \\
\text { Identification }\end{array}$ & $\begin{array}{l}\text { To analyze the user needs and specification of user demands when they access } \\
\text { the smart mHealthcare services the requirement of application-layer } \\
\text { integration and compatibility must be fulfilled. } \\
\text { The demands of the end-user and stakeholders are ensured by the HCI smart } \\
\text { mHealthcare design of the device related to the application layer services } \\
\text { users and device used. Other requirements may be extracted from legacy UI, } \\
\text { research, interviews, observations, and pre and past case report. }\end{array}$ \\
\hline $\begin{array}{l}\text { Systematic Plan User- } \\
\text { Centric Design }\end{array}$ & $\begin{array}{l}\text { For the UCD, the smart mHealthcare plan includes the analysis and } \\
\text { observations of the user environment and device. This manages the user } \\
\text { activities and history. }\end{array}$ \\
\hline
\end{tabular}

The simulation of the user environment or prototyping helps to predict the possible usability errors and interaction scenario of the user to a device to explore anomalous conditions, as shown in Fig. 3 and indicated in Tab. 2. The alert system is beneficial in the form of both sound and visual or both. This behavior is critical when the battery level is low. However, the HCI designer incorporates the measures to remind the user of the low state of the battery and to prevent the disabling of such alarms. The user and HCPs relationships demand a considerable insight into the device design. The user experience has a big impact on the devices ' design and 
UI. Hence, the designer acknowledges all the needs of the user and HCPs. The mHealthcare design method and steps should support the relationship of the HCPs and end users. The HCPs are the first and foremost group that utilizes the mHealthcare devices and gains experience, as shown in Fig. 3. The HCPs having prior knowledge of usability and design information architecture might perform well with the device, as described in Tab. 2. However, HCPs at different levels of training and expertise might require different UIs within the same device. The application supports the end-user requirements and feasible design in terms of HCI mHealthcare UI. Under the circumstances, the end-user can recognize the design and buttons and easily comprehend information at the time of rapid response.

HCPs must be ensured in a warning and possess control regarding the conditions and changes in inpatient data. However, HCPs and end-users fully comprehend and identify the design specifications as described in Tab. 2.

Nevertheless, the smart HCI mHealthcare design, specifying the needs and strategies of different Usercentric designs, include the end-user distinguishing requirements, in which HCI designers resolve conflicts arising between the HCPs and UCD or end-user usability. The usability and feasibility of the HCI smart design must meet the needs of the stakeholders, as described in Tab. 2. The end-user must not be mistaken with the customers. The end-user might not have the knowledge or technical education about the device and design or oftentimes are not the ones who buy such medical devices. The HCI smart designer incorporates the knowledge of the environment where the device will be used and who the users are, as shown in Fig. 3. The usability with robust results and good HCI UIs is not enough; mHealthcare devices must be durable and sustain long-term stability. This provides legacy support and long life to medical devices, as shown in Fig. 3. The design solution production, related to workflow, task, and content of the information architecture, results in optimized and smart HCI architecture. This result is the outcome of the previous phases of the iterative design. HCI smart mHealthcare plan includes the analysis and observations of the user environment and device. This manages the user activities and history, as described in Tab. 2. The medical devices` life cycle is long and its adoption is slow.

The HCI smart mHealthcare designer leverage previous workflows and legacy structures from user history and integration with the new environment. This helps with the understanding of the users` perspective of usability and design interface. Furthermore, this information optimizes the design and reduces the users 'error as, shown in Fig. 3. The systematic plan of UCD, based on the history of the user, optimizes the mental models and re-usability of the systems according to the user's satisfaction level, as described in Tab. 2. It supports the stable transition of new medical devices and the usability of new smart mHealthcare devices.

Time is precious and invaluable when dealing with life and health care provisions. The timely response and concurrency should be ensured at each layer and phase. In real-time data realization, the sensor layer must diminish the latency level in both availability and being measured value. Then, the communication layer plays an important role in dealing with real-time data constraints in emergency rooms or emergencies. After the communication layer, the data integration layer should provide transparency and precision in aggregation and relevancy of specific information from different sources, as shown in Figs. 2 and 3. The timestamps and data frequency indicators might be used to indicate to the user and HCPs about the timeliness and concurrency constraints at the application layer level, as shown in Tab. 2 .

In HCI smart mHealthcare design, privacy and personalization are the main needs that should be taken into great consideration. The smart mHealthcare device intelligently recognizes and saves the users` characteristics, preferences, and habitual activities. However, the device may personalize and customize the UI according to saved information, knowledge, preferences, and capabilities of the user. The HCI smart mHealthcare designer can develop better HCI design through the consideration of all provided human factors. It provides better UX (user experience) and better UI. 


\section{Conclusion}

In this paper, an architecture for intelligent mHealthcare is proposed which uses HCI (Human-Computer Interaction) based design principles to enhance the usefulness and usability of the devices and help improve experience of both the HCPs and the patients. These design principles allow the support for the User-Centric Design (UCD) for smart mHealthcare models. The proposed architecture enhances the 4-layered architecture proposed in the literature and provides an improved intelligent patient health monitoring system, which has become more critical in remote health care. The 5-layered model comprises the additional AI layer for intelligent patient situation assessment and multi-modal communication with the application layer, data integration layer, communication layer, and sensing layer. The success of the intelligent mHealthcare requires HCI design principles focusing upon the ease-of-use (including user experience) and safety, alarms, and error resistant displays of the end-user. User and HCPs relationship and personalization are incorporated along with integration with legacy workflows and security. Also, the design of intelligent virtual assistants for communicating critical patient care is crucial. Moreover, an important factor is improving the overall user experience, for both the patient and HCPs. The intelligent mHealthcare system with an actuation layer, that can administer remote care (injecting the drug, providing CPR, even surgeries), will be more convenient and smart, but it remains as future work since it requires more confidence in these technologie. In addition, it will also need more HCI based smart methodology development.

Funding Statement: The authors express their gratitude to the ministry of education and the Deanship of Scientific Research of Najran University, Kingdom of Saudi Arabia, for financial and technical support under code number NU/ESCI/17/107.

Conflicts of Interest: The authors declare that they have no conflicts of interest to report regarding the present study.

\section{References}

[1] C. Doukas and I. Maglogiannis, "Bringing IoT and cloud computing towards pervasive healthcare," in 6th Int. Conf. on Innovative Mobile and Internet Services in Ubiquitous Computing (IMIS'12). Palermo, Italy, pp. 922-926, 2012.

[2] M. Hassanalieragh, A. Page, T. Soyata, G. Sharma, M. Aktas et al., "Health monitoring and management using Internet-of-Things (IoT) sensing with cloud-based processing: Opportunities and challenges," in 2015 IEEE Int. Conf. on Services Computing, New York, NY, USA, pp. 285-292, 2015.

[3] S. A. Solangi, K. Khoumbati and D. N. Hakro, "Multi-hop optimization in wireless sensor networks using GA," Sindh Journal of Information and Communication Technology, vol. 2, no. 3, pp. 1-5, 2018.

[4] K. Khoumbati, S. A. Solangi, Z. Bhatti and D. N. Hakro, "Optimal route planning by genetic algorithm for wireless sensor networks," in Int. Conf. on Information Science and Communication Technology, Karachi, Pakistan, pp. 4-7, 2020.

[5] S. A. Solangi, D. N. Hakro, I. A. Lashari, K. R. Khoumbati, Z. A. Bhutto et al., "Genetic algorithm applications in wireless sensor networks: A review," International Journal of Management Sciences and Business Research, vol. 1, no. 4, pp. 152-166, 2017.

[6] S. A. Solangi, K. Khoumbati and D. N. Hakro, "Multi-hop optimization in wireless sensor networks using genetic algorithm," Sindh Journal of Information and Communication Technology, vol. 3, no. 4, pp. 193-197, 2019.

[7] A. A. Shah, S. A. Solangi and A. A. Shah, "Cloud computing for bio-informatics: A pakistani perspective," in Third National Conf. on Emerging Trends In Bioinformatics \& Biosciences, Mansehra, KP, Pakistan, pp. 3-5, 2019.

[8] S. Poslad, Ubiquitous System: Challenges and Outlook in Ubiquitous Computing: Smart Devices, Environments and Interactions. John Wiley \& Sons Inc., pp. 421-454, 2009, 978-0-470-03560-3 
[9] F. Alagoz, A. C. Valdez, W. Wilkowska, M. Ziefle, S. Dorner et al., "From cloud computing to mobile internet, from user focus to culture and hedonism: The crucible of mobile health care and wellness applications," in 5th Int. Conf. on Pervasive Computing and Applications (PerCom'10), Maribor, Slovenia, pp. 38-45, 2010.

[10] A. Holzinger, "From cloud computing to mobile internet, from user focus to culture and hedonism: the crucible of mobile health care and wellness applications," in 5th Int. Conf. on Pervasive Computing and Applications (PerCom'10), Maribor, Slovenia, pp. 38-45, 2010.

[11] P. Liu, S. Fels, N. Wes and M. Görges, "Human computer interaction design for mobile devices based on a smart healthcare architecture," Advances in Computers and Software Engineering: Reviews, vol. 2, pp. 99-131, 2019.

[12] J. B. Bartholomew, J. Agley, J. Carlson, K. Lay and D. Tidd, "Interprofessional development case study of an SBIRT web-based app for education and practice," Journal of Technology in Human Services, vol. 39, pp. 1-18, 2021.

[13] H. Fulford, "Exploring the use of mobile information and communication technology by people with mood disorders, and their health and social care professionals," Journal of Mental Health Nursing, vol. 28, no. 6, pp. 1268-1277, 2019.

[14] R. Robinson, “App revolution: Avoiding the app trap," PharmaVOICE, vol. 14, no. 2, pp. 24-28, 2014.

[15] J. L. Martin, B. J. Norris, E. Murphy and J. A. Crowe, "Medical device development: The challenge for ergonomics," Applied Ergonomics, vol. 39, no. 3, pp. 271-283, 2008.

[16] A. G. Money, J. Barnett, J. Kuljis, M. P. Craven, J. L. Martin et al., "The role of the user within the medical device design and development process: Medical device manufacturers' perspectives," BMC Medical Informatics and Decision Making, vol. 11, no. 15, pp. 1-12, 2011.

[17] C. J. Vincent, Y. Li and A. Blandford, "Integration of human factors and ergonomics during medical device design and development: It's all about communication," Applied Ergonomics, vol. 45, no. 3, pp. 413-419, 2014.

[18] N. Bui and M. Zorzi, "Health care applications: A solution based on the Internet of Things," in 4th Int. Sym. on Applied Sciences in Biomedical and Communication Technologies (ISABEL'11), Barcelona, Spain, pp. 1-5, 2011.

[19] R. Istepanian and B. Woodward, "M-health: Fundamentals and Applications," in Institute of Electrical and Electronics Engineers Inc. (IEEE), pp. 1-388, 2016, 978-1-118-49698-5

[20] N. L. Bragazzi, "From P0 to P6 medicine, a model of highly participatory, narrative, interactive, 'augmented' medicine: Some considerations on Salvatore Iaconesi's clinical story, Patient Prefer," Adherence, vol. 7, pp. 353-359, 2013.

[21] V. Fanos, "10 p pediatrics: Notes for the future," Journal of Pediatric and Neonatal Individualized Medicine, vol. 5, no. 2, pp. e050101-e050101, 2016.

[22] C. Röcker and A. Maeder, "User-centered design of smart healthcare applications," Electronic Journal of Health Informatics, vol. 6, no. 2, pp. e11-e11, 2011.

[23] A. Solanas, C. Patsakis, M. Conti, I. S. Vlachos, Ramos et al., "Smart health: A context-aware health paradigm within smart cities," IEEE Communications Magazine, vol. 52, no. 8, pp. 583-586, 2014.

[24] Market Research Report Multiple Trends in Mobile Health (mHealth) Industry, "Market Reports World," (Accessed 7 May 2021), 2017. [Online]. Available at: https:/www.marketreportsworld.com/.

[25] F. Firouzi, A. Rahmani, K. Mankodiya and M. Badaroglu, "Internet-of things and big data for smarter healthcare: From device to architecture, applications and analytics," Future Generation Computer Systems, vol. 78, pp. 583-586, 2018.

[26] B. Martínez-Pérez, I. de la Torre-Díez and M. López-Coronado, "Mobile health applications for the most prevalent conditions by the world health organization: Review and analysis," Journal of Medical Internet Research, vol. 15, no. 6, pp. e120-e120, 2013.

[27] J. Lee, "Smart health: Concepts and status of ubiquitous health with smartphone," in Proc. of the Int. Conf. on ICT Convergence (ICTC'11), Seoul, South Korea, pp. 388-389, 2011.

[28] E. Kartsakli, A. S. Lalos, A. Antonopoulos, S. Tennina and M. D. Renzo, "A survey on M2M systems for mHealth: A wireless communications perspective," Sensors, vol. 14, no. 10, pp. 18009-18052, 2014.

[29] M. M. Islam, M. A. Razzaque, M. M. Hassan, W. N. Ismail and B. Song, "Mobile cloud-based big healthcare data processing in smart cities," IEEE Access, vol. 5, pp. 11887-11899, 2017. 
[30] E. Kartsakli, A. Antonopoulos, A. S. Lalos, S. Tennina, D. Renzo et al., "Reliable MAC design for ambient assisted living: Moving the coordination to the cloud," IEEE Communications Magazine, vol. 53, no. 1, pp. 78-86, 2015.

[31] A. S. Lalos, A. Antonopoulos, E. Kartsakli, Di Renzo, S. Tenninaet et al., "Rlnc-aided cooperative compressed sensing for energy efficient vital signal telemonitoring," IEEE Transactions on Wireless Communications, vol. 14, no. 7, pp. 3685-3699, 2015.

[32] R. Chaudhary, A. Jindal, G. S. Aujla, N. Kumar, A. K. Das et al., "Lscsh: Lattice-based secure cryptosystem for smart healthcare in smart cities environment," IEEE Communications Magazine, vol. 56, no. 1, pp. 24-32, 2018.

[33] K. Stawarz and A. L. Cox, "Designing for health behavior change: HCI research alone is not enough," in Proc. of the Workshop: Crossing HCI and Health: Advancing Health and Wellness Technology Research in Home and Community Settings (CHI'15), Seoul, Korea, pp. 1-4, 2015.

[34] E. S. Poole, "HCI and mobile health interventions: How human-computer interaction can contribute to successful mobile health interventions," Translational Behavioral Medicine, vol. 3, no. 4, pp. 402-405, 2013.

[35] D. Pal, S. Funilkul, N. Charoenkitkarn and P. Kanthamanon, "Internet-of-things and smart homes for elderly healthcare: An end user perspective," IEEE Access, vol. 6, pp. 10483-10496, 2018.

[36] A. Blandford, J. Gibbs, N. Newhouse, O. Perski, A. Singh et al., "Seven lessons for interdisciplinary research on interactive digital health interventions," Digital Health, vol. 4, pp. 1-13, 2018.

[37] J. Reason, "Human Error," Cambridge University Press, pp. 1-3728,1990, ISBN 978-0-429-13484-5

[38] T. Webb and S. Dayal, "Building the wall: Addressing cybersecurity risks in medical devices in the U.S.A. and Australia," Computer Law \& Security Review, vol. 33, no. 4, pp. 559-563, 2017.

[39] R. J. Fairbanks and R. L. Wears, "Hazards with medical devices: The role of design," Annals of Emergency Medicine, vol. 52, no. 5, pp. 519-521, 2008.

[40] S. Hyland and J. Senders, "Integrating human factors engineering into medication safety at ISMP Canada Using Human Factors Engineering to Improve Patient Safety," in Problem Solving on the Front Line, Library of Congress Control, pp. 145-167, 2010, 978-1-59940-526-1

[41] A. Shaikh, M. Saleh, Y. Asiri, A. Sulaiman and H. Alshahrani, "Tele-COVID: A telemedicine SOA-based architectural design for COVID-19 patients," Computers, Materials \& Continua, vol. 67, no. 1, pp. 549-576, 2021.

[42] M. Alsulami and A. Shaikh, "The information-seeking problem in human-technology interaction," International Journal of Advanced Computer Science and Applications, vol. 7, no. 5, pp. 584-590, 2016.

[43] A. Shaikh, "The impact of SOA on a system design for a telemedicine healthcare system," Network Modeling Analysis in Health Informatics and Bioinformatics, vol. 4, no. 15, pp. 1-16, 2015.

[44] M. Deakin, "Smart cities: Governing, modelling and analysing the transition," Routledge, 1st edition, pp. 1-232, 2014, ISBN 978-0-415-65819-5

[45] L. Catarinucci, D. de Donno, L. Mainetti, L. Palano, L. Patrono et al., "An IoT-aware architecture for smart healthcare systems," IEEE Internet of Things Journal, vol. 2, no. 6, pp. 515-526, 2015.

[46] P. Liu and Z. Peng, "China's smart city pilots: A progress report," Computer (Long Beach Calif.), vol. 47, no. 10, pp. 72-81, 2014.

[47] G. Manogaran, C. Thota, D. Lopez and R. Sundarasekar, "Big data security intelligence for healthcare industry 4.0," in Cybersecurity for Industry 4.0: Analysis for Design and Manufacturing. Springer, pp.103-126, 2017, 978-3-319-50660-9

[48] S. Sakr and A. Elgammal, "Towards a comprehensive data analytics framework for smart healthcare services," Big Data Research, vol. 4, pp. 44-58, 2016.

[49] M. C. Domingo, "An overview of the internet of things for people with disabilities," Journal of Network and Computer Applications, vol. 35, no. 2, pp. 584-596, 2012.

[50] D. Gil, A. Ferrández, H. Mora-Mora and J. Peral, "Internet of things: A review of surveys based on context aware intelligent services," Sensors, vol. 16, no. 7, pp. 1-23, 2016.

[51] S. El Jaouhari, A. Bouabdallah, J. M. Bonnin and T. Lemlouma, "Toward a smart health-care architecture using WebRTC and WoT, " in Recent Advances in Information Systems and Technologies. Vol. 3, Springer, pp. 531-540, 2017. 
[52] M. Görges, C. L. Petersen, G. A. Dumont and J. M. Ansermino, "Using machine-to-machine/"Internet of Things' communication to simplify medical device information exchange," in Int. Conf. on the Internet of Things (IoT'14), Cambridge, MA, USA, pp. 49-54, 2014.

[53] S. Saeed, A. Shaikh, S. M. Raza and M. A. Memon, "Impact of data mining techniques to analyze health care data," Journal of Medical Imaging and Health Informatics, vol. 8, no. 5, pp. 674-682, 2018.

[54] I. Chiuchisan, H. Costin and O. Geman, "Adopting the internet of things technologies in health care systems," in Proc. of the Int. Conf. and Exposition on Electrical and Power Engineering (EPE'14), Romania Iasi, Romania, pp. 532-535, 2014.

[55] C. C. Chen and Y. F. Chang, "Smart healthcare environment: Design with rfid technology and performance evaluation," Journal of Medical and Biological Engineering, vol. 33, pp. 427-432, 2013.

[56] H. Demirkan, "A smart healthcare systems framework," IT Professional, vol. 15, no. 5, pp. 38-45, 2013.

[57] M. J. Deen, "Information and communications technologies for elderly ubiquitous healthcare in a smart home," Personal and Ubiquitous Computing, vol. 19, no. 3-4, pp. 573-599, 2015.

[58] R. C. Park, H. Jung, D. K. Shin, G. J. Kim and K. H. Yoon, "M2M-based smart health service for human UI/UX using motion recognition," Cluster Computing, vol. 18, no. 1, pp. 221-232, 2015.

[59] D. Saha and A. Mukherjee, "Pervasive computing: A paradigm for the 21st century," Computer (Long Beach Calif.), vol. 36, no. 3, pp. 25-31, 2003.

[60] J. Casselman, N. Onopa and L. Khansa, "Wearable healthcare: Lessons from the past and a peek into the future," Telematics and Informatics, vol. 34, no. 7, pp. 1011-1023, 2017.

[61] M. Gastaldi, "Integration of mobile, big data, sensors, and social media: Impact on daily life and business," in ISTAfrica Conf., Mauritius, pp. 1-10, 2014.

[62] A. Shaikh, "NoMadCar: The Interactive Design using Human Computer Interaction Techniques," Journal of Advanced Computer Science and Technology Research, vol. 5, no. 1, pp. 1-15, 2015.

[63] W. Jordon, "Usability 101: Introduction to Usability," Taylor and Francis, pp. 1-111, 1998, 0-7484-0762-6

[64] A. Shaikh, "An interactive design using human computer interaction for autonomous vehicles," International Journal of Engineering Trends and Technology (IJETT), vol. 10, no. 1, pp. 160-172, 2020.

[65] N. G. Leveson and C. S. Turner, "An investigation of the Therac-25 accidents," Computer (Long Beach Calif.), vol. 26, no. 7, pp. 18-41, 1993.

[66] J. D. Gould and C. Lewis, "Designing for usability: Key principles and what designers think," Communications of The ACM, vol. 28, no. 3, pp. 300-311, 1985.

[67] S. Z. Lowry, P. Abbott, M. C. Gibbons, R. North, E. S. Patterson et al., "Technical evaluation, testing, and validation of the usability of electronic health records," National Institute of Standards and Technology, pp. 65-87, 2012.

[68] S. Saeed, A. Shaikh, M. A. Memon, M. Q. Saleem and S. M. R. Naqvi, "Assessment of brain tumor due to the usage of MATLAB performance," Journal of Medical Imaging and Health Informatics, vol. 7, pp. 1454-1460, 2017.

[69] ECRI Institute, “Top 10 health technology hazards for 2012," Health Devices, vol. 40, no. 11, pp. 1-18, 2011.

[70] P. Potter, L. Wolf, S. Boxerman, D. Grayson and J. Sledge, "Understanding the cognitive work of nursing in the acute care environment," Journal of Nursing Administration, vol. 35, no. 7-8, pp. 327-335, 2005.

[71] S. Sendelbach and M. F. Care, "Alarm fatigue a patient safety concern," AACN Advanced Critical Care, vol. 24, no. 4, pp. 378-386, 2013.

[72] J. Watson, A. Kinstler, W. P. Vidonish, M. Wagner, L. Lin et al., "Impact of noise on nurses in pediatric intensive care units," American Journal of Critical Care, vol. 24, no. 5, pp. 377-384, 2015.

[73] D. A. Norman, "Categorization of action slips," Psychological Review, vol. 88, no. 1, pp. 1-15, 1981. 